\title{
Health Care Costs in a Cohort of HIV-Infected U.S. Veterans Receiving Regimens Containing Tenofovir Disoproxil Fumarate/Emtricitabine
}

\author{
Richard E. Nelson, PhD; Junjie Ma, MS; Jacob Crook, MS; Kristin Knippenberg, MFA; \\ Heather Nyman, PharmD; Damemarie Paul, MPH; Stephen Esker, PharmD, AAHIVP; \\ and Joanne LaFleur, PharmD, MSPH
}

\begin{abstract}
BACKGROUND: Tenofovir disoproxil fumarate (TDF), a key component in many human immunodeficiency virus (HIV) treatment regimens, is associated with increased renal and bone toxicities. The contributions of such toxicities to treatment costs, as well as the relative differences in treatment costs for various TDF/emtricitabine (FTC) regimens, remains unexplored.

OBJECTIVE: To estimate and compare mean overall and renal- and bonespecific costs, including total, inpatient, outpatient, and pharmacy costs in patients treated with TDF/FTC+efavirenz (EFV) compared with several nonEFV-containing TDF/FTC regimens.
\end{abstract}

METHODS: We conducted a national cohort study of treatment-naive HIVinfected U.S. veterans who initiated treatment from 2003 to 2015 with TDF/ FTC in combination with EFV, elvitegravir/cobicistat, rilpivirine, or ritonavirboosted protease inhibitors (atazanavir, darunavir, or lopinavir). Outcomes of interest were quarterly total, inpatient, outpatient, and pharmacy costs using data from the Veterans Health Administration (VHA) electronic medical record and Managerial Cost Accounting System (an activity-based accounting system that allocates VHA expenditures to patient encounters). We controlled for measured confounders using inverse probability of treatment (IPT) weights and assessed differences using standardized mean differences (SMDs). For comparisons where SMDs exceeded 0.1 after IPT weighting, we used the more conservative matching weights in sensitivity analyses. For hypothesis testing, we compared IPT-adjusted differences in quarterly costs between treatment groups using Mann-Whitney U-tests and generalized estimating equation (GEE) regression models.

RESULTS: Of 33,048 HIV-positive veterans, 7,222 met eligibility criteria, including 4,172 TDF/FTC+EFV recipients; mean (SD) age of the cohort was 50.0 (10.0) years; $96.7 \%$ were male; $60.1 \%$ were black; and $30.1 \%$ were white. Quarterly periods of exposure to EFV-containing regimens were 22,499 and of exposure to non-EFV-containing regimens were 11,633. After IPT weighting, absolute SMDs were $<0.1$ except for a few covariates in the rilpivirine comparison. The per-patient adjusted mean total quarterly costs were $\$ 7,145$ for EFV versus $\$ 8,726$ for non-EFV $(P<0.001$; Mann-Whitney $U$-test) and the per-patient adjusted mean difference in total quarterly costs was $\$ 1,419$ lower for EFV versus all non-EFV combined $(P<0.001$; GEE model). Corresponding values for outpatient costs $(\$ 2,656$ vs. $\$ 2,942$; $P<0.001$; difference, $-\$ 254 ; P=0.001)$, inpatient costs $(\$ 2,009$ vs. $\$ 2,614 ; P<0.001)$, radiology costs ( $\$ 213$ vs. $\$ 276 ; P<0.001)$, and pharmacy costs ( $\$ 2,480$ vs. $\$ 3,170 ; P<0.001$; difference, $-\$ 600 ; P<0.001)$ were all lower for EFV versus all non-EFV combined. Findings based on matching weights were qualitatively similar. Contributions of renal and bone costs to the total costs of treatment were very small, ranging between $\$ 52$ and \$94 per patient per quarter for renal outcomes and between \$6 and $\$ 114$ for bone outcomes.

CONCLUSIONS: Among 7,222 HIV-treated veterans over an average follow-up of 1.2 years per patient, those patients receiving TDF/FTC + EFV had lower overall health care costs compared with those receiving non-EFV regimens.

J Manag Care Spec Pharm. 2018;24(10):1052-66

Copyright $\odot 2018$, Academy of Managed Care Pharmacy. All rights reserved.

\section{What is already known about this subject}

Efavirenz-based tenofovir disoproxil fumarate (TDF)/emtricitabine (FTC) regimens are associated with lower bone and renal toxicity compared with other TDF/FTC regimens.

A reduced risk of renal and bone adverse events should be associated with lower health care costs with EFV compared with other third agents.

\section{What this study adds}

This study estimated cost burden for various TDF/FTC regimens based on differences in the third agent and estimated the extent to which differences in renal and bone toxicity affected those costs. Among 7,222 HIV-treated veterans over an average follow-up of 1.2 years per patient, those patients receiving TDF/FTC+EFV had lower overall health care costs compared with those receiving non-EFV regimens.

$\mathrm{T}$ enofovir disoproxil fumarate (TDF)/emtricitabine (FTC) is the most commonly used antiretroviral therapy (ART) backbone for treating human immunodeficiency virus (HIV) worldwide. ${ }^{1}$ However, epidemiologic studies have shown increased renal and bone toxicities associated with TDF, contributing to guideline recommendations that TDF should be used with caution or avoided in patients with renal disease and osteoporosis. ${ }^{1}$ However, switching patients who are currently stable on TDF/FTC-based regimens, which continue to be recommended in World Health Organization guidelines, ${ }^{2}$ should be approached cautiously, particularly as newer evidence has identified that the renal and bone adverse effects may be attenuated by pairing TDF/FTC with certain third agents. For example, TDF/FTC regimens that include efavirenz (EFV) have been associated with lower rates of adverse renal and bone outcomes compared with the addition of other third agents. EFV, a non-nucleoside reverse transcriptase inhibitor (NNRTI), has been associated with lower risks of both proteinuria and chronic kidney disease (CKD) compared with tenofovir, a nucleoside reverse transcriptase inhibitor ${ }^{3}$; lower TDF-associated renal impairment in ART combinations that included EFV compared with nevirapine, an $\mathrm{NNRTI}^{4}$; and preserved bone mineral density (BMD) compared with atazanavir (ATV), a protease inhibitor (PI). . $^{5}$ 
Estimates of the real-world economic burden of TDF/FTCassociated adverse events (AEs), including renal and bone outcomes, are lacking in the existing literature. Nadkarni et al. (2015) reported that the incidence of hospitalizations for acute kidney injury in patients with HIV doubled from 2002 to 2010, which they attributed to the aging of the HIV population and increases in comorbidities; however, ART effects on acute kidney injury were not examined..$^{9}$ Magoni et al. (2011), who reported the effect of HIV on renal disease in the Italian health care system, also did not evaluate ART effects. ${ }^{10}$ Koenig et al. (2010) evaluated the cost-effectiveness of monitoring for renal insufficiency in Haitian HIV patients on ART and found it to be cost prohibitive with negligible clinical benefits in a resource-limited setting. ${ }^{11}$ In a cost-effectiveness modeling study, Walensky et al. (2016) determined that the reduced renal and bone toxicity of tenofovir alafenamide justified up to a $\$ 1,000 /$ year increase in cost for tenofovir alafenamide versus $\mathrm{TDF},{ }^{12}$ largely due to an assumed high cost for hemodialysis of $\$ 87,000$ based upon an estimated rate of hemodialysis of $25 \%$ in those discontinuing TDF. However, this assumption is not supported by evidence from the Data collection on Adverse events of Anti-HIV Drugs (D:A:D) study demonstrating that end-stage renal disease (ESRD) in patients with HIV infection over a median follow-up of 6 years was associated with diabetes, hypertension, baseline estimated glomerular filtration rate (eGFR), smoking, and current CD4 cell count rather than past or current exposure to TDF. ${ }^{13,14}$

The real-world cost differences associated with treatment and maintenance of HIV patients across TDF/FTC regimens in combination with different third agents in a U.S. setting remain unknown. The relative contributions of renal and bone adverse outcomes to these costs are also unknown, both on a per-patient basis and on a health-system basis. Given that TDF/ FTC regimens that include EFV have demonstrated relatively lower rates of adverse renal and bone outcomes compared with other TDF/FTC regimens, ${ }^{3,4,6-8}$ a reduced risk of these adverse outcomes with EFV may also be associated with lower health care costs with EFV compared to other third agents.

Our primary objectives in this analysis were to (a) estimate the adjusted mean total, outpatient, and pharmacy costs of HIV treatment with TDF/FTC in combination with several common base agents and (b) compare adjusted mean differences in total, outpatient, and pharmacy costs. We did this in a cohort of treatment-naive U.S. veterans initiating treatment with TDF/FTC plus EFV, elvitegravir/cobicistat (EVG/c), rilpivirine (RPV), or boosted PIs. In a supplemental analysis, we also examined the renal- and bone-specific components of costs and use of the Veterans Affairs (VA) health care system.

\section{Methods}

\section{Study Design and Datasets}

This was a nationwide, historical cohort study of treatmentnaive, HIV-infected patients initiating ART in the Veterans
Health Administration (VHA). The VHA is the largest integrated health care delivery network in the United States, with more than 150 VHA hospitals and 850 outpatient clinics. All datasets were hosted in the VA Veterans Informatics and Computing Infrastructure (VINCI) environment. VINCI is a new initiative to improve researchers' access to VHA data and to facilitate the analysis of that data with integrated tools while ensuring patient privacy and data security in a secure workspace. The University of Utah Institutional Review Board and the Salt Lake City VA Health Care System Office of Research and Development approved this study.

\section{Study Population}

Inclusion Criteria. All treatment-naive HIV-infected adults diagnosed with HIV infection from January 1, 2003, through December 31, 2015, and who received an ART regimen of interest and who had at least 1 VA outpatient encounter 6 months or more before starting their ART regimen were included. HIV infection was identified using diagnosis codes from the International Classification of Diseases, Ninth/Tenth Revisions, Clinical Modification (ICD-9/10-CM). The index date was defined as the date the patient received the first ART prescription (any time between January 1, 2003, and December 31, 2015), comprising TDF/FTC with (a) EFV (as EFV/TDF/FTC in fixed-dose combination [FDC] or EFV plus TDF/FTC in FDC); (b) RPV (as RPV/TDF/FTC in FDC or RPV plus TDF/FTC in FDC); (c) EVG/c (as EVG/c/TDF/FTC in FDC); or (d) ritonavir (RTV)-boosted ATV, lopinavir (LPV), or darunavir (DRV; as boosted-PI plus TDF/FTC in FDC).

Exclusion Criteria. Patients were excluded if they had a pharmacy claim for any ART agent in the pre-index period, defined as the 6-month period before the index date. To further ensure each patient was antiretroviral naive and not receiving continuing ART from previous treatment outside the VHA, we used a validated method for excluding patients who had most likely received previous treatment. ${ }^{20}$ This method excluded patients if (a) the index regimen was a "salvage" regimen (i.e., composed of both a PI and NNRTI or composed of $\geq 5$ agents) or (b) any HIV RNA level $<500$ copies per $\mathrm{mL}$ during the preindex period, which suggested prior antiretroviral exposure. ${ }^{20}$ We also excluded patients who did not have an inpatient or outpatient encounter at least 6 months before the index date to ensure that we captured patients with a pattern of receiving routine care within the VHA.

Follow-up. Patients were followed until they discontinued the ART regimen of interest or until the end of the study period (December 31, 2015), whichever was sooner. We defined discontinuation as a gap of more than 30 days for any 1 of the medications in a patient's ART regimen. The date of discontinuation was the first day of such a gap. 


\section{Study Variables}

Outcomes/Dependent Variables. Our primary outcome variables included quarterly total, outpatient, inpatient, and pharmacy costs during the period following the index date up to discontinuation of the regimen of interest or the end of the study period. These quarterly costs were calculated by summing all VHA health care costs for inpatient, outpatient, and pharmacy services provided in each quarter after the index date based on data available via the Managerial Cost Accounting System (an activity-based accounting system that allocates VHA expenditures to patient encounters). Quarterly use outcomes were assessed by counting frequencies of outpatient visits and numbers of inpatient days for hospital stays in the VHA during the quarter in which they occurred.

To contrast with the overall costs and use previously described, in supplemental analyses, we calculated quarterly bone- and renal-specific costs and use by summing all VHA health care costs and use associated with an ICD-9/10-CM or Current Procedural Terminology, 4th Edition (CPT-4) code for bone disease (i.e., osteoporosis diagnosis or a vertebral, hip, or wrist fracture) or renal disease (i.e., proteinuria, CKD, or ESRD, including renal transplant and dialysis). All codes for bone and renal disease are available via Web links 1 and 2, respectively.

We used cost per patient-quarter as our unit of measure because duration of follow-up varied substantially between individuals. All cost outcomes were converted to 2015 U.S. dollars (USD) using the personal consumption expenditures index published by the U.S. Bureau of Economic Analysis. ${ }^{24}$

Exposures/Independent Variables. The exposure of interest was TDF/FTC in combination with EFV compared with TDF/ FTC in combination with (a) RPV, (b) EVG/c, or (c) boosted PIs. These 3 TDF/FTC-based FDC regimens were ascertained based on pharmacy claims information.

Covariates/Control Variables. Patient characteristics with a known or theoretical association with exposures and outcomes were identified based on literature search and expert consensus. Identified covariates measured during the pre-index period included demographics (i.e., age, race, body mass index, marital status, sex), disease severity measures (i.e., CD4 count, viral load), measures of comorbid disease (i.e., bone disease, albumin, eGFR, and comorbid conditions), drug exposures (i.e., methadone, proton pump inhibitors, bisphosphonates), and cost. These were assessed in the 6 months before each patient's index date and were used to control for potential confounding.

Bone disease during the pre-index period was defined as a diagnosis of osteoporosis/osteopenia by ICD-9/10-CM diagnosis codes, CPT-4 codes, or classification by BMD test result. Data on patient BMD T-scores were derived from narrative records using a validated natural language processing (NLP) technique. ${ }^{21}$ NLP can be used to extract clinical data from radiology reports, clinicians' progress notes, and other text reports. This validated technique has been applied in previous studies to extract BMD T-scores from radiology reports. ${ }^{21-23}$ In validation, an examination of test characteristics for the tool revealed that the NLP tool for BMD from radiology reports had an overall accuracy of $92.8 \%$ for the anatomic site at which the BMD was measured, $92.8 \%$ for the value of the BMD T-score, and $90.4 \%$ for the matching the correct BMD T-score value with the correct anatomical site. ${ }^{21}$

\section{Statistical Analysis}

Outcomes. We reported patient characteristics during the pre-index period using descriptive statistics and assessed differences between the groups using standardized mean differences (SMDs); absolute SMDs $>0.1$ were considered meaningful differences. ${ }^{25}$ To control for confounding by indication and selection bias, we estimated inverse probability of treatment (IPT) weights, ${ }^{26}$ an extension of the propensity score, which represents the conditional probability of receiving the treatment as predicted by covariates described above. IPT weights were calculated from a propensity score generated using logistic regression that estimated the probability that each patient would initiate TDF/FTC plus EFV. ${ }^{26}$ In the event that covariate balance using IPT weights was not achieved for any of the comparisons, we conducted a sensitivity analysis using matching weights. ${ }^{27}$ For our multivariable models, we included a category for "missing" data. ${ }^{28}$

We compared adjusted costs and use outcomes using IPTweighted Mann-Whitney U-tests to compare distributions for all pair-wise comparisons versus EFV. We also conducted IPT-weighted generalized estimating equation (GEE) regression models to estimate the adjusted mean differences in quarterly overall outpatient, pharmacy, and total costs and outpatient use associated with exposure to each regimen of interest in the weighted cohort. We identified the most appropriate distribution for the GEE model by using the modified Park test. ${ }^{29}$ In addition, we used the method of recycled predictions to generate estimates of the marginal effect of each exposure on cost and use in the original scale. ${ }^{30}$ The relative change in cost associated with EFV-based regimens versus each non-EFV regimen was expressed as the expenditure rate ratio (ERR).

Supplemental Analysis. For adjusted mean differences of overall inpatient and radiology costs and use, as well as for adjusted mean differences of any renal and bone cost or use, we used a 2-part model ${ }^{31}$ to calculate the marginal effect associated with each exposure, conditional upon having that cost or use. Specifically, part 1 of the model assessed the odds of having a positive cost (logistic regression) and part 2 assessed the ERR for EFV versus other regimens conditional on having a positive cost (IPT-weighted GEE). 


\section{FIGURE 1 Eligibility Criteria for Patient Selection}

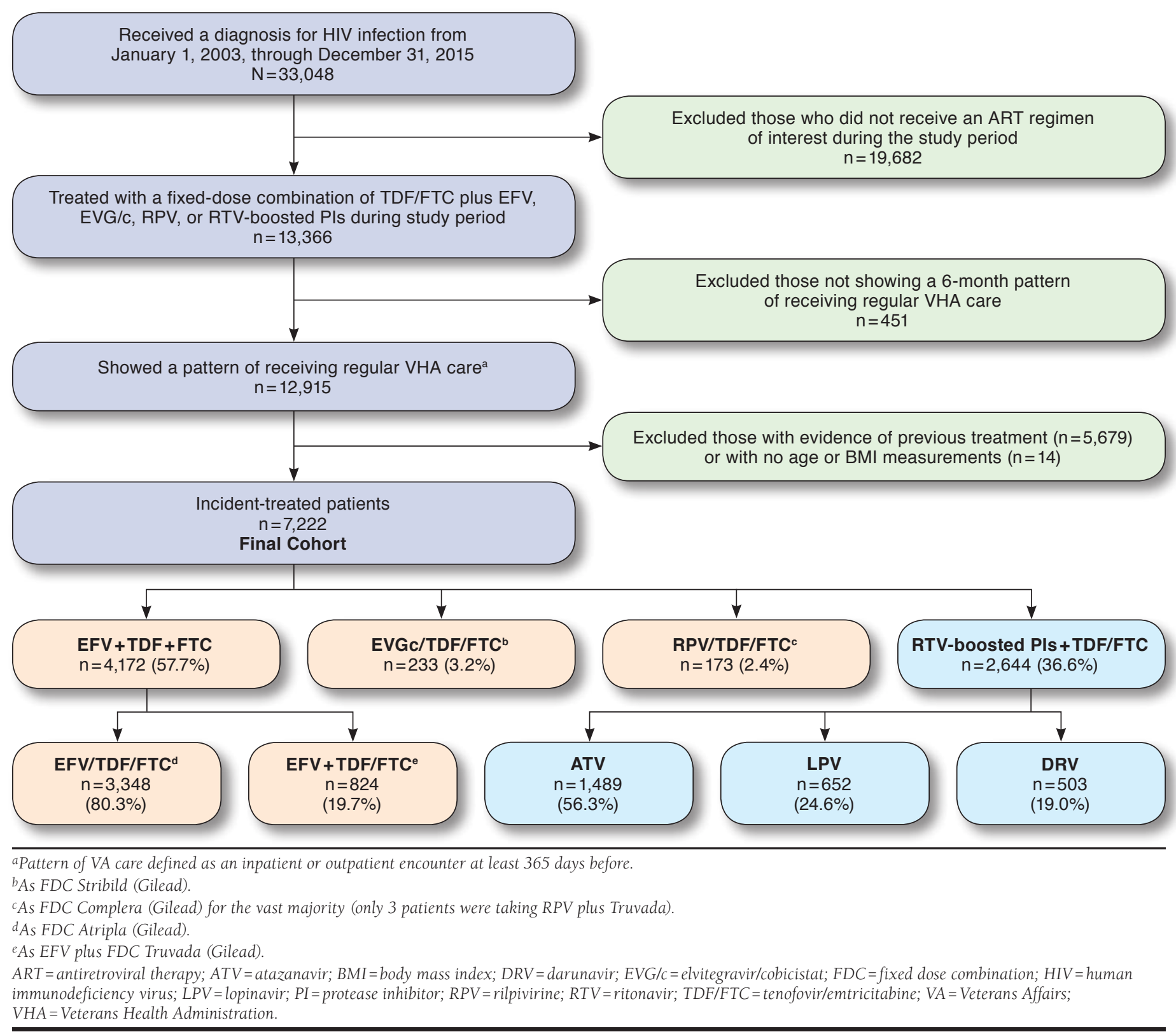

\section{Results}

\section{Patient Characteristics}

Of 33,048 HIV-positive veterans, 13,366 received ART regimens of interest while 7,222 were treatment naive (EFV, $\mathrm{n}=4,172$; non-EFV, $\mathrm{n}=3,050$; Figure 1). The average age of patients in the cohort was 50 years, and $96.5 \%$ were male. These patients were followed for an average (standard deviation [SD]) of 13.0 (19.2) months, including 15.0 (21.1), 9.8 (7.6), 9.9 (10.5), and 10.5 (16.7) months for EFV+TDF/FTC,
EVG/c/TDF/FTC, RPV/TDF/FTC, and RTV-boosted PIs + TDF/ FTC, respectively. Corresponding median (interquartile range) values were 5.0 (2.0-14.2), 5.7 (2.1-17.9), 4.6 (2.1-10.4), 5.9 (2.8-13.4), and 4.2 (1.8-10.7) months. Patients treated with an EFV regimen had lower mean annual total costs $(\$ 18,000$ during the pre-index period) compared with other non-EFV regimens (ranging from $\$ 20,000$ for non-EFV and boosted PIs to $\$ 33,000$ for EVG/c; Table 1$)$. 


\section{TABLE 1 Unadjusted Characteristics in the Pre-Index Period Among HIV-Infected Veterans Receiving Initial} ART with Differing TDF/FTC-Containing Regimens

\begin{tabular}{|c|c|c|c|c|c|c|c|c|c|c|}
\hline \multirow[b]{2}{*}{ Characteristic $^{a}$} & \multicolumn{10}{|c|}{ Initial ART Containing TDF/FTC Plus } \\
\hline & \multicolumn{2}{|c|}{$\begin{array}{c}\text { EFV } \\
(n=4,172)\end{array}$} & \multicolumn{2}{|c|}{$\begin{array}{l}\text { Non-EFVb } \\
(\mathrm{n}=3,050)\end{array}$} & \multicolumn{2}{|c|}{$\begin{array}{c}\text { EVG/c } \\
(n=233)\end{array}$} & \multicolumn{2}{|c|}{$\underset{(n=173)}{R P V}$} & \multicolumn{2}{|c|}{$\begin{array}{l}\text { RTV-Boosted PI } \\
\quad(\mathrm{n}=2,644)\end{array}$} \\
\hline Age, years, mean \pm SD & \multicolumn{2}{|c|}{$50 \pm 10$} & \multicolumn{2}{|c|}{$49 \pm 9.8$} & \multicolumn{2}{|c|}{$49 \pm 13$} & \multicolumn{2}{|c|}{$48 \pm 13$} & \multicolumn{2}{|c|}{$50 \pm 9.3$} \\
\hline Male & 4,033 & $(96.7)$ & 2,925 & (95.9) & 222 & (95.3) & 163 & $(94.2)$ & 2,540 & (96.1) \\
\hline $\mathrm{BMI}, \mathrm{kg} / \mathrm{m}^{2}$, mean $\pm \mathrm{SD}$ & \multicolumn{2}{|c|}{$26 \pm 5.0$} & \multicolumn{2}{|c|}{$25 \pm 5.1$} & \multicolumn{2}{|c|}{$26 \pm 5.6$} & \multicolumn{2}{|c|}{$27 \pm 5.3$} & \multicolumn{2}{|c|}{$25 \pm 5.1$} \\
\hline Married, n (\%) & 340 & $(8.1)$ & 224 & $(7.3)$ & 26 & $(11.2)$ & 20 & $(11.6)$ & 178 & $(6.7)$ \\
\hline \multicolumn{11}{|l|}{ Race, n (\%) } \\
\hline White & 1,256 & $(30.1)$ & 923 & (30.3) & 74 & (31.8) & 48 & $(27.7)$ & 801 & $(30.3)$ \\
\hline Black & 2,509 & $(60.1)$ & 1,773 & $(58.1)$ & 124 & $(53.2)$ & 106 & $(61.3)$ & 1,543 & $(58.4)$ \\
\hline Hispanic & 262 & $(6.3)$ & 209 & $(6.9)$ & 19 & $(8.2)$ & 10 & $(5.8)$ & 180 & $(6.8)$ \\
\hline Asian & 34 & $(0.8)$ & 28 & $(0.9)$ & 5 & $(2.1)$ & 2 & $(1.2)$ & 21 & $(0.8)$ \\
\hline Other & 22 & $(0.5)$ & 28 & $(0.9)$ & 2 & (0.9) & 2 & (1.2) & 24 & (0.9) \\
\hline \multicolumn{11}{|l|}{ CD4+ count, cells/mm ${ }^{3}$} \\
\hline$<200$ & 1,296 & $(31.1)$ & 1,110 & $(36.4)$ & 60 & $(25.8)$ & 23 & (13.3) & 1,027 & $(38.8)$ \\
\hline $200-299$ & 704 & (16.9) & 481 & (15.8) & 33 & $(14.2)$ & 30 & (17.3) & 418 & (15.8) \\
\hline $300-399$ & 639 & (15.3) & 346 & (11.3) & 35 & (15.0) & 11 & $(6.4)$ & 300 & (11.3) \\
\hline $400-499$ & 404 & $(9.7)$ & 224 & $(7.3)$ & 24 & $(10.3)$ & 33 & $(19.1)$ & 167 & $(6.3)$ \\
\hline$\geq 500$ & 597 & (14.3) & 433 & $(14.2)$ & 61 & $(26.2)$ & 65 & $(37.6)$ & 307 & (11.6) \\
\hline Missing & 532 & (12.8) & 456 & $(15.0)$ & 20 & $(8.6)$ & 11 & $(6.4)$ & 425 & (16.1) \\
\hline \multicolumn{11}{|l|}{ HIV viral load, copies/mL } \\
\hline$<10,000$ & 1,196 & $(28.7)$ & 959 & (31.4) & 66 & $(28.3)$ & 70 & $(40.5)$ & 823 & $(31.1)$ \\
\hline $10,000-100,000$ & 1,445 & (34.6) & 949 & $(31.1)$ & 78 & $(33.5)$ & 67 & $(38.7)$ & 804 & $(30.4)$ \\
\hline$>100,000$ & 922 & (22.1) & 691 & $(22.7)$ & 61 & $(26.2)$ & 15 & $(8.7)$ & 615 & (23.3) \\
\hline Missing & 609 & (14.6) & 451 & (14.8) & 28 & $(12.0)$ & 21 & $(12.1)$ & 402 & (15.2) \\
\hline Chronic kidney disease $^{c}$ & 284 & $(6.8)$ & 172 & $(5.6)$ & 16 & $(6.9)$ & 10 & $(5.8)$ & 146 & $(5.5)$ \\
\hline \multicolumn{11}{|l|}{$\mathrm{eGFR}, \mathrm{mL} / \mathrm{min} / 1.73 \mathrm{~m}^{2}$} \\
\hline Stage $1, \geq 90$ & 2,205 & $(52.9)$ & 1,564 & (51.3) & 143 & $(61.4)$ & 110 & (63.6) & 1,311 & $(49.6)$ \\
\hline Stage 2, 60-89 & 1,382 & (33.1) & 997 & (32.7) & 74 & (31.8) & 54 & $(31.2)$ & 869 & (32.9) \\
\hline Stage 3, 30-59 & 164 & (3.9) & 124 & $(4.1)$ & 3 & (1.3) & 4 & $(2.3)$ & 117 & $(4.4)$ \\
\hline Stage 4, 15-29 & 5 & $(0.1)$ & 7 & $(0.2)$ & 0 & $(0.0)$ & 0 & $(0.0)$ & 7 & $(0.3)$ \\
\hline Stage $5,<15$ & 39 & $(0.9)$ & 53 & $(1.7)$ & 0 & $(0.0)$ & 0 & $(0.0)$ & 53 & $(2.0)$ \\
\hline Missing & 377 & (9.0) & 305 & $(10.0)$ & 13 & (5.6) & 5 & (2.9) & 287 & (10.9) \\
\hline
\end{tabular}

After IPT weighting, all absolute SMDs were $<0.10$ except for 5 covariates in the RPV comparison, which were between 0.1 and 0.2 ; these included age, viral load, CKD, cardiovascular disease, and diabetes (Figure 2). After weighting with matching weights, all SMDs were $<0.1$ (data not shown), meaning the results were qualitatively similar.

\section{Outcomes}

Adjusted Mean Total, Inpatient, Outpatient, and Pharmacy Costs. Adjusted mean overall costs were significantly lower for EFV versus all other regimens for every type of cost, including total, outpatient, inpatient, radiology, and pharmacy (all $P<0.001$; Table 2). Adjusted mean total quarterly cost associated with EFV was $\$ 7,145$, compared with $\$ 8,532$ with PI-based regimens (the most commonly prescribed drug class of the non-EFV regimens), $\$ 8,726$ with all non-EFV regimens combined, \$11,258 with RPV-based regimens, and \$11,728 with EVG/c-based regimens. In the GEE regression, the adjusted mean differences of total quarterly costs were $\$ 1,419$ $(P<0.001), \$ 4,488(P=0.012), \$ 3,372(P=0.042)$, and $\$ 1,303$ $(P<0.001)$ lower for EFV regimens versus non-EFV overall, EVG/c, RPV, and PI regimens, respectively (Table 3). Total costs and pharmacy costs were consistently statistically significant, with pharmacy costs being most greatly reduced for EFV in comparison to EVG/c (a reduction of $\$ 1,876 ; P<0.001$ ). For EFV regimens versus non-EFV regimens, outpatient costs were $\$ 254(P=0.001)$ lower, while pharmacy costs were $\$ 600$ lower $(P<0.001)$. Compared with all other regimens of interest, ERRs indicated that EFV regimens were associated with significantly lower quarterly expenditure rates for outpatient (9.0\%-17.4\% lower), pharmacy (20.4\%-46.1\% lower), or total costs (15.7\%39.5\% lower; Table 3). Unadjusted means and standard deviations are presented in Appendix A (available in online article). 


\section{Health Care Costs in a Cohort of HIV-Infected U.S. Veterans Receiving Regimens Containing Tenofovir Disoproxil Fumarate/Emtricitabine}

\section{TABLE 1 Unadjusted Characteristics in the Pre-Index Period Among HIV-Infected Veterans Receiving Initial}

ART with Differing TDF/FTC-Containing Regimens (continued)

\begin{tabular}{|c|c|c|c|c|c|c|}
\hline \multirow[b]{2}{*}{ Characteristic $^{a}$} & \multicolumn{6}{|c|}{ Initial ART Containing TDF/FTC Plus } \\
\hline & $\begin{array}{c}\text { EFV } \\
(n=4,172)\end{array}$ & $\begin{array}{l}\text { Non-EFVb } \\
(\mathbf{n}=3,050)\end{array}$ & $\begin{array}{c}\text { EVG/c } \\
(\mathrm{n}=233)\end{array}$ & $\begin{array}{c}\text { RPV } \\
(\mathbf{n}=173)\end{array}$ & \multicolumn{2}{|c|}{$\begin{array}{l}\text { RTV-Boosted PI } \\
\quad(\mathrm{n}=2,644)\end{array}$} \\
\hline \multicolumn{7}{|l|}{ Comorbidities, n (\%) } \\
\hline $\mathrm{CAD} / \mathrm{CVD}$ & $(10.9)$ & $(9.2)$ & $(12.0)$ & $(7.5)$ & 241 & $(9.1)$ \\
\hline Heart failure & $(3.2)$ & $(2.0)$ & $(2.1)$ & $(1.2)$ & 55 & $(2.1)$ \\
\hline Dyslipidemia & $(16.1)$ & $(13.7)$ & $(21.5)$ & $(16.8)$ & 340 & $(12.9)$ \\
\hline Hypertension & $(37.0)$ & $(30.2)$ & $(35.6)$ & $(38.2)$ & 772 & $(29.2)$ \\
\hline Diabetes mellitus & $(13.6)$ & $(11.9)$ & $(13.3)$ & $(11.0)$ & 313 & $(11.8)$ \\
\hline End-stage renal disease $^{\mathrm{d}}$ & $(0.4)$ & $(0.4)$ & $(0.4)$ & $(0.0)$ & 12 & $(0.5)$ \\
\hline Viral hepatitis & $(27.1)$ & $(29.8)$ & $(25.3)$ & $(24.9)$ & 807 & $(30.5)$ \\
\hline Tuberculosis & $(1.2)$ & $(1.5)$ & $(0.4)$ & $(1.2)$ & 43 & (1.6) \\
\hline Psychiatric disorder & $(36.3)$ & $(45.8)$ & $(51.1)$ & $(60.7)$ & 1,172 & $(44.3)$ \\
\hline Depression & $(23.3)$ & $(29.0)$ & $(35.6)$ & $(39.3)$ & 733 & $(27.7)$ \\
\hline Schizophrenia & $(3.4)$ & $(6.4)$ & $(5.2)$ & $(5.2)$ & 173 & $(6.5)$ \\
\hline Bipolar disorder & $(15.5)$ & $(21.4)$ & $(26.2)$ & $(36.4)$ & 530 & $(20.0)$ \\
\hline Psychosis & $(5.6)$ & $(10.2)$ & $(11.2)$ & $(11.0)$ & 265 & $(10.0)$ \\
\hline Posttraumatic stress disorder & $(8.9)$ & $(12.6)$ & $(16.7)$ & $(30.6)$ & 292 & $(11.0)$ \\
\hline Bone disease $e^{e}$ & $(0.9)$ & $(0.9)$ & $(2.1)$ & $(1.2)$ & 20 & $(0.8)$ \\
\hline Tobacco use $\mathrm{f}^{\mathrm{f}}$ & $(30.9)$ & $(30.5)$ & $(30.0)$ & $(45.1)$ & 781 & $(29.5)$ \\
\hline Alcohol abuse & $(22.9)$ & $(24.0)$ & $(25.3)$ & $(29.5)$ & 623 & $(23.6)$ \\
\hline \multicolumn{7}{|l|}{ Medications, n (\%) } \\
\hline Methadone & $(1.2)$ & $(2.4)$ & $(0.9)$ & $(1.2)$ & 68 & $(2.6)$ \\
\hline Proton pump inhibitors & $(28.5)$ & $(23.7)$ & $(24.5)$ & $(13.9)$ & 643 & $(24.3)$ \\
\hline Bisphosphonates & $\begin{array}{ll}21 & (0.5) \\
\end{array}$ & $18 \quad(0.6)$ & $0 \quad(0.0)$ & $1 \quad(0.6)$ & 17 & $(0.6)$ \\
\hline \multicolumn{7}{|c|}{ 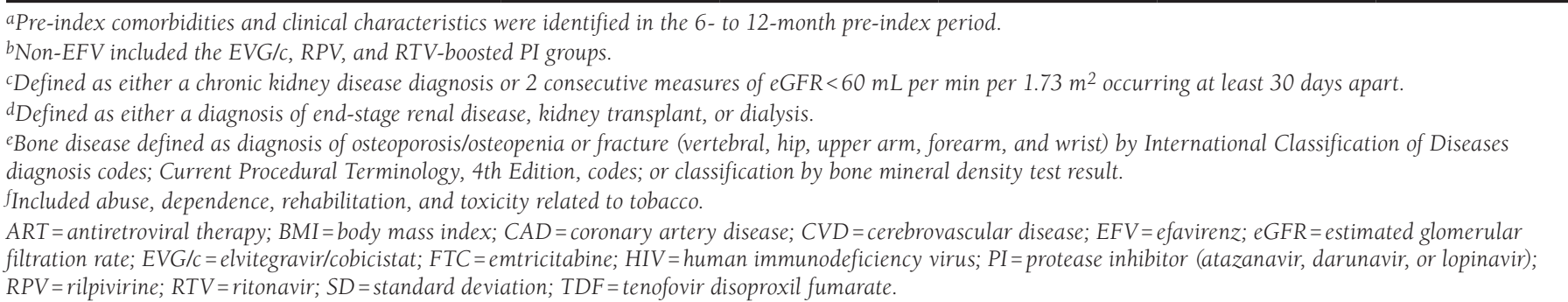 } \\
\hline
\end{tabular}

Adjusted Mean Renal- and Bone-Specific Costs. Adjusted mean renal and bone costs were only a small fraction of the overall costs associated with each exposure. Quarterly renal costs ranged from $\$ 29$ on the RPV regimen (vs. \$11,258 total costs on RPV) to $\$ 88$ on the EVG/c regimen (vs. $\$ 11,728$ total costs on EVG/c). Bone costs ranged from $\$ 6$ on the EVG/c regimen (vs. $\$ 11,728$ total costs) to $\$ 114$ on the RPV regimen (vs. $\$ 11,258$ total costs). Differences were statistically significant for many comparisons, with some comparisons favoring EFV and others favoring comparators (Table 2).

Inpatient and Outpatient Utilization. Overall outpatient and inpatient use on EFV regimens were also significantly lower compared with all non-EFV regimens as well as each of the individual regimens (Table 2). The overall total quarterly outpatient encounters were lower with EFV compared with all non-EFV regimens; mean differences were $-2.39(P<0.001)$,
$-5.25(P=0.006),-9.53(P=0.050 ; 95 \%$ confidence interval $[C I]=-19.08-0.02)$, and $-2.40(P=0.001)$ versus non-EFV, EVG/c, RPV, and PI regimens, respectively (Table 3).

Notably, renal and bone use followed the trends of overall use, being generally lower for patients on EFV than on any other regimen and significantly so only in comparisons with EVG/c and RPV regimens (Table 2).

Supplemental 2-Part Models. Because very few patients had zero outpatient or pharmacy costs in the overall analyses, 2-part models were not undertaken for these outcomes in that analysis. In overall 2-part models, the odds of having a positive inpatient or radiology cost were significantly lower for patients on EFV compared with those on PI-based regimens (part 1 models: Appendix B, available in online article). However, among patients who incurred an overall inpatient or radiology cost, none of the mean differences between regimens for such costs 


\section{Hypertension \\ Index year 2017 \\ Index year 2010 \\ Index year 2009 \\ CD4 300-399 cells $/ \mathrm{mm}^{3}$ \\ Proton pump inhibitor Age, years \\ Index year 2008 \\ CD4 400-499 cells $/ \mathrm{mm}^{3}$}

HIV viral load 10,000-100,000 copies/mL Heart failure Index year 2011

Dyslipidemia CAD/CVD

Diabetes mellitus Chronic kidney disease Body mass index, $\mathrm{kg} / \mathrm{m}^{2}$ Black race Male sex eGFR stage $1, \geq 90 \mathrm{~mL} / \mathrm{min} / 1.74 \mathrm{~m}^{2}$ CD4 200-299 cells $/ \mathrm{mm}^{3}$ Married Bone disease Tobacco use eGFR stage 2, $60-90 \mathrm{~mL} / \mathrm{min} / 1.73 \mathrm{~m}^{2}$ CD4 $\geq 500$ cells $/ \mathrm{mm}^{3}$ Index year 2012 White race HIV viral load missing End-stage renal disesae eGFR stage $3,30-59 \mathrm{~mL} / \mathrm{min} / 1.73 \mathrm{~m}^{2}$ Asian race Bisphosphonate HIV viral load $\geq 100,000$ copies $/ \mathrm{mL}$ Index year 2004 Tuberculosis Hispanic race Alcohol use eGFR stage $4,15-29 \mathrm{~mL} / \mathrm{min} / 1.73 \mathrm{~m}^{2}$ eGFR missing Other raceIndex year 2006 Missing race Viral hepatitis HIV viral load $<10,000$ copies $/ \mathrm{mL}$ Pre-index cost CD4 missing eGFR stage $5,<15 \mathrm{~mL} / \mathrm{min} / 1.73 \mathrm{~m}^{2}$ Index year 2013 Methadone CD4 $<200$ cells/mm3 Posttraumatic stress disorderDepression Schizophrenia Bipolar Psychosis Index year 2005 Index year 2014 Psychiatric disorder Index year 2015

$$
-0.3
$$

Overrepresented in non-EFV

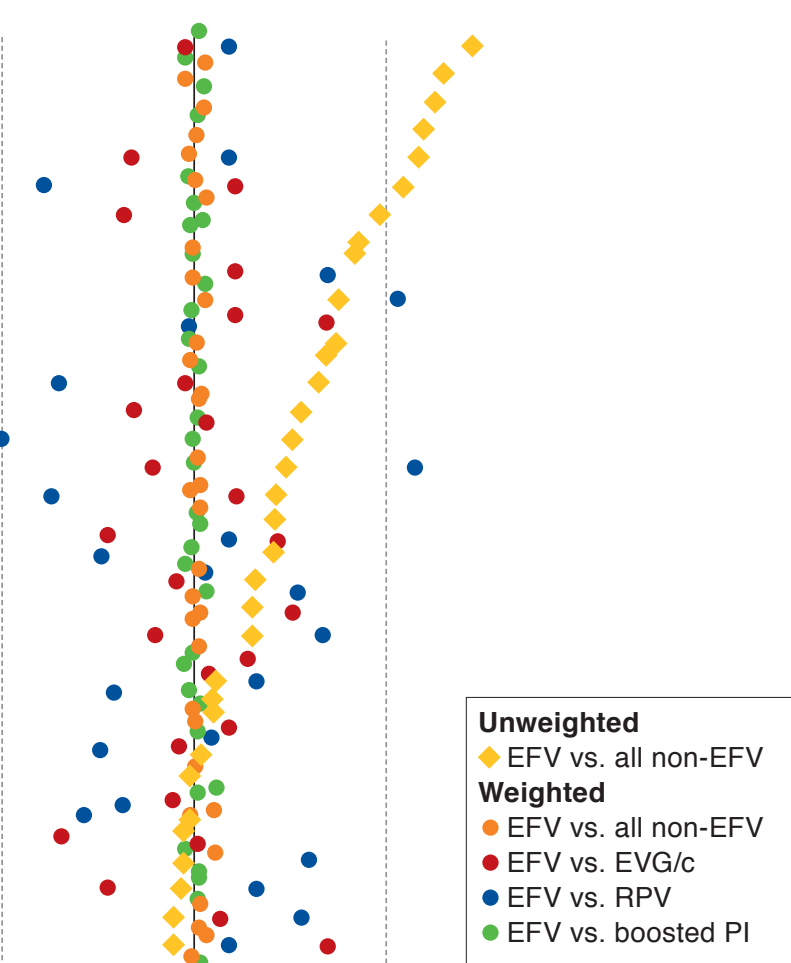

$C A D=$ coronary artery disease; $C V D=$ cerebrovascular disease; $E F V=$ efavirenz; $e G F R=$ estimated glomerular filtration rate; EVG/c =elvitegravir/cobicistat; FTC = emtricitabine; HIV=human immunodeficiency virus; IPT = inverse probability of treatment; PI= protease inhibitor (atazanavir, darunavir, or lopinavir); $R P V=$ rilpivirine; $R T V=$ ritonavir; $T D F=$ tenofovir disoproxil fumarate. 


\section{Health Care Costs in a Cohort of HIV-Infected U.S. Veterans Receiving Regimens Containing Tenofovir Disoproxil Fumarate/Emtricitabine}

\begin{tabular}{|c|c|c|c|c|c|c|c|c|c|c|}
\hline & \multicolumn{2}{|c|}{ EFV } & $\begin{array}{c}\text { All } \\
\text { Non-EFV }\end{array}$ & $\begin{array}{c}P \\
\text { Value }^{b}\end{array}$ & EVG/c & $\begin{array}{c}P \\
\text { Value }^{\mathrm{b}}\end{array}$ & RPV & $\begin{array}{c}P \\
\text { Value }^{b}\end{array}$ & $\begin{array}{c}\text { RTV-Boosted } \\
\text { PI } \\
\end{array}$ & $\begin{array}{c}P \\
\text { Value }^{\mathrm{b}}\end{array}$ \\
\hline Number & \multicolumn{2}{|c|}{4,172} & 3,050 & & 233 & & 173 & & 2,644 & \\
\hline Quarters & \multicolumn{2}{|c|}{5.6} & 4.0 & & 3.0 & & 3.9 & & 4.0 & \\
\hline Patient-quarters & \multicolumn{2}{|c|}{23,189} & 12,073 & & 707 & & 667 & & 10,699 & \\
\hline \multicolumn{11}{|l|}{ Overall } \\
\hline \multicolumn{11}{|l|}{ VA cost, \$ } \\
\hline Outpatient & 2,656 & $(26)$ & 2,942 & $<0.001$ & $3,068 \quad(179)$ & $<0.001$ & $3,410 \quad(287)$ & $<0.001$ & 3,036 & $<0.001$ \\
\hline Inpatient & 2,009 & $(81)$ & $2,614 \quad(161)$ & $<0.001$ & $4,253(1,509)$ & $<0.001$ & $4,399(1,441)$ & $<0.001$ & 2,583 & $<0.001$ \\
\hline Radiology & 213 & (7) & $276 \quad(23)$ & $<0.001$ & $258 \quad(35)$ & $<0.001$ & $285 \quad(61)$ & $<0.001$ & $286 \quad(26)$ & $<0.001$ \\
\hline Pharmacy & 2,480 & (39) & $3,170 \quad(48)$ & $<0.001$ & $4,407 \quad(181)$ & $<0.001$ & $3,866 \quad(271)$ & $<0.001$ & 3,223 & $<0.001$ \\
\hline Total cost & 7,145 & (97) & $8,726 \quad(180)$ & $<0.001$ & $11,728(1,585)$ & $<0.001$ & $11,258(1,499)$ & $<0.001$ & 8,532 & $<0.001$ \\
\hline \multicolumn{11}{|l|}{ VA use } \\
\hline Outpatient encounters & 18.3 & $(0.4)$ & $(0.3)$ & $<0.001$ & $23.1 \quad(17.6)$ & $<0.001$ & $(2.5)$ & $<0.001$ & $(0.3)$ & $<0.001$ \\
\hline Inpatient days & 1.5 & $(0.1)$ & $(0.1)$ & $<0.001$ & $1.7 \quad(0.3)$ & $<0.001$ & $(0.4)$ & $<0.001$ & $(0.1)$ & $<0.001$ \\
\hline \multicolumn{11}{|l|}{ Renal } \\
\hline \multicolumn{11}{|l|}{ VA cost, $\$$} \\
\hline Outpatient & 9 & (1) & $(2)$ & 0.248 & $(5)$ & $<0.001$ & (8) & $<0.001$ & $(2)$ & 0.239 \\
\hline Inpatient & 40 & $(10)$ & (9) & 0.009 & $(57)$ & $<0.001$ & (13) & 0.082 & (9) & 0.014 \\
\hline Total & 49 & (11) & (9) & 0.35 & $(61)$ & $<0.001$ & (16) & $<0.001$ & (10) & 0.110 \\
\hline \multicolumn{11}{|l|}{ VA use } \\
\hline Outpatient encounters & \multicolumn{2}{|c|}{$0.054(0.004)$} & $0.062(0.007)$ & 0.977 & $0.130(0.084)$ & 0.007 & $0.125(0.036)$ & $<0.001$ & $0.059(0.007)$ & 0.933 \\
\hline Inpatient days & 0.087 & $.009)$ & $0.087(0.012)$ & 0.182 & $0.150(0.045)$ & $<0.001$ & $0.429(0.196)$ & $<0.001$ & $0.073(0.010)$ & 0.408 \\
\hline Bone & & & & & & & & & & \\
\hline VA cost, $\$$ & & & & & & & & & & \\
\hline Outpatient & 10 & (3) & $(2)$ & 0.172 & (3) & $<0.001$ & $(23)$ & 0.603 & $(2)$ & $<0.001$ \\
\hline Inpatient & 20 & $(8)$ & $(7)$ & 0.542 & $(0)$ & 0.008 & $(75)$ & $<0.001$ & $(7)$ & 0.008 \\
\hline Total & 29 & (9) & $(7)$ & 0.004 & (3) & $<0.001$ & $(81)$ & $<0.001$ & $(7)$ & $<0.001$ \\
\hline VA use & & & & & & & & & & \\
\hline Outpatient encounters & 0.014 & $.003)$ & $0.018(0.003)$ & 0.023 & $0.020(0.009)$ & 0.079 & $0.058(0.032)$ & 0.002 & $0.016(0.002)$ & 0.105 \\
\hline Inpatient days & 0.014 & $.005)$ & $0.03(0.009)$ & 0.001 & $0.071(0.046)$ & 0.029 & $0.017(0.015)$ & $<0.001$ & $0.031(0.012)$ & 0.002 \\
\hline $\begin{array}{l}{ }^{a} \text { Costs are in } 2015 \text { U.S. doll } \\
\text { bP value versus EFV. P value } \\
E F V=\text { efavirenz; } E V G / c=e l v \\
R T V=\text { ritonavir; } S D=\text { standa }\end{array}$ & . & cistat & $\begin{array}{l}=\text { inverse proba } \\
\text { rans Affairs. }\end{array}$ & 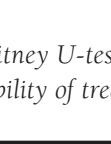 & ment: $P I=$ protea & 1. & azanavir, daru & 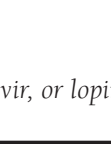 & ir); $R P V=$ rilp & \\
\hline
\end{tabular}

were significant after adjustment (part 2 models: Appendix B). Similarly, the odds of having positive inpatient use were lower with EFV versus all non-EFV regimens; however, among these patients with positive inpatient use, there were no significant adjusted mean differences in inpatient use (Appendix B).

In renal 2-part models, the odds of having a positive pharmacy cost was significantly higher versus EVG/c; however, among these patients with a positive cost, the adjusted mean difference in cost was not significant (Appendix B). The odds of having positive inpatient costs, inpatient use, and outpatient use were significantly lower with EFV versus a number of comparators; however, among these patients with positive costs, significant adjusted mean differences in cost were found for only 2 comparisons (EFV vs. PI for inpatient cost, difference $\$ 10,254, P=0.041$; and EFV vs. RPV for outpatient encounters, difference 0.69, $P=0.014$; Appendix B).
In bone 2-part models, the odds of having a positive inpatient cost was significantly lower versus RPV; however, among these patients with a positive cost, the adjusted mean difference in cost was not significant (Appendix B). No other bone outcomes showed significantly different odds of having a positive cost.

\section{Discussion}

We identified the cost and use effects of different third agents in TDF/FTC ART regimens within a nationwide VHA setting. Adjusted mean quarterly overall costs and use consistently and significantly favored EFV over boosted PIs, EVG/c, and RPV in combination with TDF/FTC, including total, inpatient, outpatient, pharmacy, and radiology costs. We found that patients receiving EFV regimens had quarterly costs that were $\$ 1,419$ (95\% CI $=\$ 843-\$ 1,994)$ lower than those receiving all non-EFV 


\section{Health Care Costs in a Cohort of HIV-Infected U.S. Veterans Receiving Regimens Containing Tenofovir Disoproxil Fumarate/Emtricitabine}

\section{TABLE 3 Adjusted Results from IPT-Weighted GEE Model for Quarterly Overall Costs and Utilizations}

\begin{tabular}{|c|c|c|c|c|c|}
\hline & PQs & ERR $(95 \%$ CI) & $P$ Value & $\begin{array}{c}\text { Mean Difference per PQ } \\
\$(95 \% \mathrm{CI})\end{array}$ & $P$ Value \\
\hline \multicolumn{6}{|c|}{ EFV vs. non-EFV } \\
\hline \multicolumn{6}{|c|}{ Cost } \\
\hline Outpatient & 35,262 & $(0.860-0.963)$ & 0.001 & $-254 \quad(-408--100)$ & 0.001 \\
\hline Pharmacy & 35,262 & $(0.750-0.842)$ & $<0.001$ & $-600 \quad(-752--447)$ & $<0.001$ \\
\hline Total & 35,262 & $(0.780-0.897)$ & $<0.001$ & $-1,419 \quad(-1,994--843)$ & $<0.001$ \\
\hline \multicolumn{6}{|l|}{ Utilization } \\
\hline Outpatient & 35,262 & $(0.821-0.945)$ & $<0.001$ & $(-3.72--1.06)$ & $<0.001$ \\
\hline \multicolumn{6}{|l|}{ EFV vs. EVG/c } \\
\hline \multicolumn{6}{|l|}{ Cost } \\
\hline Outpatient & 23,896 & $(0.703-0.970)$ & 0.020 & $-520 \quad(-985--54)$ & 0.029 \\
\hline Pharmacy & 23,896 & $(0.481-0.603)$ & $<0.001$ & $-1,876 \quad(-2,299--1,452)$ & $<0.001$ \\
\hline Total & 23,896 & $(0.433-0.829)$ & 0.002 & $-4,488 \quad(-7,987--990)$ & 0.012 \\
\hline \multicolumn{6}{|l|}{ Utilization } \\
\hline Outpatient & 23,896 & $(0.639-0.910)$ & 0.003 & $-5.25 \quad(-8.99--1.52)$ & 0.006 \\
\hline \multicolumn{6}{|l|}{ EFV vs. RPV } \\
\hline \multicolumn{6}{|l|}{ Cost } \\
\hline Outpatient & 23,856 & $(0.682-1.195)$ & 0.476 & $-270 \quad(-1,045-505)$ & 0.494 \\
\hline Pharmacy & 23,856 & $(0.555-0.750)$ & $<0.001$ & $-1,267 \quad(-1,791--742)$ & $<0.001$ \\
\hline Total & 23,856 & $(0.492-0.926)$ & 0.015 & $-3,372 \quad(-6,617--128)$ & 0.042 \\
\hline \multicolumn{6}{|l|}{ Utilization } \\
\hline Outpatient & 23,856 & $(0.448-0.923)$ & 0.017 & $-9.53(-19.08-0.02)$ & 0.050 \\
\hline \multicolumn{6}{|c|}{ EFV vs. RTV-boosted PIs } \\
\hline \multicolumn{6}{|c|}{ Cost } \\
\hline Outpatient & 33,888 & $0.898 \quad(0.847-0.953)$ & $<0.001$ & $-286 \quad(-446--126)$ & $<0.001$ \\
\hline Pharmacy & 33,888 & $0.796 \quad(0.750-0.846)$ & $<0.001$ & $-579 \quad(-736--422)$ & $<0.001$ \\
\hline Total & 33,888 & $0.846 \quad(0.790-0.906)$ & $<0.001$ & $-1,303 \quad(-1,850--756)$ & $<0.001$ \\
\hline \multicolumn{6}{|l|}{ Utilization } \\
\hline Outpatient & 33,888 & $0.878 \quad(0.816-0.945)$ & 0.001 & $(-3.77--1.03)$ & 0.001 \\
\hline
\end{tabular}

regimens combined. Furthermore, the adjusted mean differences of pharmacy costs all favored EFV over the other regimens, with pharmacy cost savings ranging from $\$ 579$ versus PIs to $\$ 1,876$ versus EVG/c. This study is the first to quantify total, inpatient, outpatient, pharmacy, and radiology costs associated with differing third agents in TDF/FTC regimens. These results shed light on the effect of ART regimens on mean quarterly cost from the patient's perspective. The overall cost advantages of EFV regimens (80.3\% EFV/FTC/TDF (Atripla, Gilead) and 29.7\% EFV+FTC/TDF (Truvada, Gilead; Figure 1) versus non-EFV regimens may be due to several possible reasons. They may be directly related to regimen cost or unmeasured concomitant medications; alternatively, they may be due to unmeasured adverse outcomes from other comorbidities exacerbated by TDF/FTC regimens.

Although renal and bone use followed the trends of overall use in the primary analysis, 2-part models, exploring renal and bone costs and use in the smaller population of patients who did have positive costs or use, did not consistently follow the overall results. Renal and bone costs were a very small proportion of overall costs, and the CIs in analysis were often wide in these 2-part analyses due to the small sample sizes. Because renal- and bone-specific costs made up such a small proportion of overall costs ( $<1 \%$ according to our data), we concluded that renal and bone disease were not major contributors to the overall cost of long-term HIV treatment in this population.

The disconnect between the overall and the renal and bone costs can likely be attributed to the rarity of renal and bone outcomes but also to other, unmeasured health care needs. HIV drug costs are the main cost for long-term HIV patients, although estimates vary from setting to setting. ${ }^{18,32}$ The cost-effectiveness analysis (CEA) conducted by Simpson et al. (2013) provides a list of other common health care needs for patients with HIV, ${ }^{18}$ gleaned from South Carolina 
Medicaid data from the early highly active antiretroviral therapy era (i.e., 2002-2003, all inflated to 2011 USD). For example, chronic opportunistic infections requiring ongoing treatment were found to cost anywhere from $\$ 5,052$ annually for cytomegalovirus colitis to $\$ 120,000$ annually for cytomegalovirus retinitis. ${ }^{18}$ Nonserious side effects priced low (from $\$ 0$ to $\$ 223$ per episode), while serious side effects (including renal failure at $\$ 7,913$ per episode) and acute opportunistic infections were more costly, ranging from $\$ 1,336$ for an episode of viral infection causing cervical cancer to $\$ 20,248$ for a histoplasmosis episode. Although the current relative costs of HIV-related events can only be approximated from this list, due to its age and the fact that it focuses primarily on health care needs of patients on PIs, we can infer the relative frequency, importance, and costliness of renal events compared with other HIV- and ART-related events such as acute and chronic infections, even if it does not provide us with real-world incidences of these needs.

Numerous CEAs that compare various ART regimens, several of which include renal AEs as model inputs, rarely feature renal or bone disease as an outcome associated with ART generally, and none with TDF/FTC specifically. ${ }^{33-35}$ One CEA by Juday et al. (2013) determined that the FDC TDF/FTC/EVG/C was not cost-effective when compared with the FDC TDF/FTC/ EFV at $\$ 100,000 / q u a l i t y$-adjusted life years; these results were very sensitive to likelihood of renal AEs. ${ }^{36}$ Ultimately, most of these analyses considered renal disease as only a contributing factor to the overall cost of HIV or ART, and only one ${ }^{12}$ considered bone disease as a contributing factor. Cumulatively, these studies and other CEAs investigating ART generally ${ }^{15-19}$ indicate a research need to analyze the economic burden of various components of HIV care associated with different TDF/ FTC-based ART regimens.

A key strength of this study can be attributed to the use of VHA datasets. The VHA is the largest integrated provider of HIV care in the United States, allowing a large sample size and to evaluate multiple subgroups. ${ }^{37}$ The majority market share, single payer, and integrated care attributes of this population enable observations to be made with robust estimates across the HIV continuum of care.

\section{Limitations}

Limitations of this study include those typical of observational studies. First, there was an increased risk of residual confounding due to unmeasured differences in patient characteristics during the pre-index period. However, IPT weighting can reliably produce 2 groups that are balanced with respect to measured confounders, allowing valid inferences about treatment differences to be made. Nonetheless, IPT weighting is unable to control for unmeasured confounders that may affect the choice of initial ART or unmeasured outcomes in the study.

Second, the costs and use captured in our study likely represent an underestimate of total costs and use within the cohort because of health care encounters and costs being limited to those occurring within the VHA; a quarter to nearly one-half of veterans receive some health care in non-VHA facilities. ${ }^{38-40}$ Studies comparing demographic and disease characteristics in populations receiving exclusive VHA versus mixed VHA/ Medicare services are conflicting; for example, 1 investigation found that patients considered at highest risk were more likely to be receiving mixed VHA and Medicare than exclusive VHA services, ${ }^{41}$ and in another investigation, patients with higher indices of socioeconomic advantage were more likely to be receiving mixed versus exclusive VHA services. ${ }^{42}$ However, given the age distribution of our VHA population, less than 5\% would have been eligible for Medicare, making it unlikely that dual health care usage affected our conclusions.

Third, because the follow-up time for patients in our cohort varied substantially, we divided the follow-up period into quarters. Alternative statistical methods exist to analyze health care costs in the presence of censoring. For example, Lin et al. (1997) proposed an approach that constructs a survivaladjusted estimator. ${ }^{43}$ Bang and Tsiatis (2000) improved on this approach by performing inverse probability weighting with survival from censoring probabilities and assuming continuous death and censoring times. ${ }^{44}$ Basu and Manning (2010) extended these methods to allow for estimation of the effect of covariates on survival effects and intensity effects separately. ${ }^{45}$ Future work should apply these methods to estimate the health care costs associated with TDF/FTC ART regimens.

Fourth, the VHA patient population has a small number of females, which limits the generalizability of the results to the wider population of patients with HIV infection, particularly concerning osteoporosis, where disease burden and treatment differ substantially between men and women. ${ }^{46}$ Finally, because the outcomes have been historically obtained rather than gathered prospectively, the lack of ascertainment of nonVHA pharmacotherapies or care limits the generalizability of these findings to non-VHA contexts.

\section{Conclusions}

We found that patients on TDF/FTC regimens with EFV had lower overall health care costs and were less likely to have inpatient admissions than those on regimens of TDF/FTC plus RPV, EVG/c, or boosted PIs. 


\section{Health Care Costs in a Cohort of HIV-Infected U.S. Veterans Receiving Regimens Containing Tenofovir Disoproxil Fumarate/Emtricitabine}

\section{Authors}

RICHARD E. NELSON, PhD, and JACOB CROOK, MS, VA Salt Lake City Health Care System, and Department of Internal Medicine, University of Utah School of Medicine, Salt Lake City. JUNJIE MA, MS; KRISTIN KNIPPENBERG, MFA; and JOANNE LAFLEUR, PharmD, MSPH, VA Salt Lake City Health Care System, and Department of Pharmacotherapy, University of Utah College of Pharmacy, Salt Lake City. HEATHER NYMAN, PharmD, Department of Pharmacotherapy, University of Utah College of Pharmacy, Salt Lake City. DAMEMARIE PAUL, MPH, and STEPHEN ESKER, PharmD, AAHIVP, Research and Development, Bristol-Myers Squibb, Plainsboro, New Jersey.

AUTHOR CORRESPONDENCE: Richard E. Nelson, PhD, Research Assistant Professor, Division of Epidemiology, University of Utah School of Medicine, 500 Foothill Blvd., Salt Lake City, UT 84148. Tel.: 801.582.1565; E-mail: richard.nelson@utah.edu.

\section{DISCLOSURES}

This study was funded by Bristol-Myers Squibb. Nelson, Ma, Crook, Knippenberg, Nyman, and LaFleur are employees of the University of Utah, which received a grant from Bristol-Myers Squibb to conduct this study. Nyman also discloses honoraria for consulting from Otsuka and for writing a book chapter from Fresenius. La Fleur reports advisory board and consulting fees from Bristol-Myers Squibb outside of this study. Paul and Esker are employees of, and own stock in, Bristol-Myers Squibb.

\section{ACKNOWLEDGMENTS}

Medical writing assistance was provided by Julian Martins of Science Communications, Springer Healthcare, which was funded by Bristol-Myers Squibb.

\section{REFERENCES}

1. Panel on Antiretroviral Guidelines for Adults and Adolescents. Guidelines for the use of antiretroviral agents in HIV-l-infected adults and adolescents. 2017. Available at: https://aidsinfo.nih.gov/guidelines/html/1/adult-and-adolescent-arv-guidelines/11/what-to-start. Accessed July 26, 2018.

2. World Health Organization. Consolidated guidelines on the use of antiretroviral drugs for treating and preventing HIV infection: recommendations for a public health approach—second edition. 2016. Available at: http:// www.who.int/hiv/pub/arv/arv-2016/en/. Accessed July 26, 2018.

3. Scherzer R, Estrella M, Li Y, et al. Association of tenofovir exposure with kidney disease risk in HIV infection. AIDS. 2012;26(7):867-75.

4. Manosuthi W, Mankatitham W, Lueangniyomkul A, et al. Renal impairment after switching from stavudine/lamivudine to tenofovir/lamivudine in NNRTI-based antiretroviral regimens. AIDS Res Ther. 2010;7:37.

5. Brown TT, Qaqish RB. Antiretroviral therapy and the prevalence of osteopenia and osteoporosis: a meta-analytic review. AIDS. 2006;20(17):2165-74.

6. McComsey GA, Kitch D, Daar ES, et al. Bone mineral density and fractures in antiretroviral-naive persons randomized to receive abacavir-lamivudine or tenofovir disoproxil fumarate-emtricitabine along with efavirenz or atazanavir-ritonavir: AIDS Clinical Trials Group A5224s, a substudy of ACTG A5202. J Infect Dis. 2011;203(12):1791-801.
7. Nkhoma ET, Rosenblatt L, Myers J, Villasis-Keever A, Coumbis J. Realworld assessment of renal and bone safety among patients with HIV infection exposed to tenofovir disoproxil fumarate-containing single-tablet regimens. PLoS One. 2016;11(12):e0166982.

8. LaFleur J, Bress A, Esker S, et al. Renal outcomes with efavirenz + tenofovir disporoxil fumarate/emtricitabine versus other tenofovir disoproxil fumarate-containing antiretroviral regimens among HIV-infected veterans: a national study. Paper presented at: 18th International Workshop on Comorbidities and Adverse Drug Reactions in HIV; September 12-13, 2016; New York, NY.

9. Nadkarni GN, Patel AA, Yacoub R, et al. The burden of dialysis-requiring acute kidney injury among hospitalized adults with HIV infection: a nationwide inpatient sample analysis. AIDS. 2015;29(9):1061-66.

10. Magoni M, Scarcella C, Vassallo F, et al. The evolving burden of HIV infection compared with other chronic diseases in northern Italy. HIV Med. 2011;12(3):129-37.

11. Koenig SP, Schackman BR, Riviere C, et al. Clinical impact and cost of monitoring for asymptomatic laboratory abnormalities among patients receiving antiretroviral therapy in a resource-poor setting. Clin Infect Dis. 2010;51(5):600-08.

12. Walensky RP, Horn TH, Paltiel AD. The Epi-TAF for tenofovir disoproxil fumarate? Clin Infect Dis. 2016;62(7):915-18.

13. Cooper RD, Wiebe N, Smith N, Keiser P, Naicker S, Tonelli M. Systematic review and meta-analysis: renal safety of tenofovir disoproxil fumarate in HIV-infected patients. Clin Infect Dis. 2010;51(5):496-505

14. Ryom L, Mocroft A, Kirk O, et al. Predictors of advanced chronic kidney disease and end-stage renal disease in HIV-positive persons. AIDS. 2014;28(2):187-99.

15. Brogan AJ, Mrus J, Hill A, Sawyer AW, Smets E. Comparative costefficacy analysis of darunavir/ritonavir and other ritonavir-boosted protease inhibitors for first-line treatment of HIV-1 infection in the United States. HIV Clin Trials. 2010;11(3):133-44.

16. Brogan AJ, Talbird SE, Cohen C. Cost-effectiveness of nucleoside reverse transcriptase inhibitor pairs in efavirenz-based regimens for treatment-naive adults with HIV infection in the United States. Value Health. 2011;14(5):657-64.

17. Broder MS, Chang EY, Bentley TG, Juday T, Uy J. Cost effectiveness of atazanavir-ritonavir versus lopinavir-ritonavir in treatment-naive human immunodeficiency virus-infected patients in the United States. J Med Econ. 2011;14(2):167-78.

18. Simpson KN, Pei PP, Moller J, et al. Lopinavir/ritonavir versus darunavir plus ritonavir for HIV infection: a cost-effectiveness analysis for the United States. Pharmacoeconomics. 2013;31(5):427-44.

19. Sweet DE, Altice FL, Cohen CJ, Vandewalle B. Cost-effectiveness of single- versus generic multiple-tablet regimens for treatment of HIV-l infection in the United States. PLoS One. 2016;11(1):e0147821.

20. Braithwaite RS, Kozal MJ, Chang CC, et al. Adherence, virological and immunological outcomes for HIV-infected veterans starting combination antiretroviral therapies. AIDS. 2007;21(12):1579-89.

21. LaFleur J, Ginter T, Curtis J, et al. A novel method for obtaining bone mineral densities from a dataset of radiology reports and clinic notes: natural language processing in a national cohort of postmenopausal veterans. Poster presented at: Annual Meeting of the American Society for Bone and Mineral Research (ASBMR); October 4-7, 2013; Baltimore, MD. Available at: http://www.asbmr.org/education/AbstractDetail?aid=99cbf7b6-f3c3-49c49984-0ba2962f1812. Accessed August 20, 2018.

22. LaFleur J, Ginter T, Hayden C, DuVall S, Adler R, Nebeker J. Natural language processing (NLP) technology for extracting bone mineral density (BMD) results from radiology reports in the Veterans Affairs (VA) healthcare system. Poster presented at: American Society of Bone and Mineral Research 2011 Annual Meeting; September 16-20, 2011; San Diego, CA. 


\section{Health Care Costs in a Cohort of HIV-Infected U.S. Veterans Receiving Regimens Containing Tenofovir Disoproxil Fumarate/Emtricitabine}

23. LaFleur J, DuVall SL, Willson T, et al. Analysis of osteoporosis treatment patterns with bisphosphonates and outcomes among postmenopausal veterans. Bone. 2015;78:174-85.

24. Bureau of Economic Analysis. Personal Consumption Expenditures Index. Database. 2017. Available at: http://www.bea.gov. Accessed July 26, 2018

25. Austin PC. Balance diagnostics for comparing the distribution of baseline covariates between treatment groups in propensity-score matched samples. Stat Med. 2009;28(25):3083-107.

26. Robins JM, Hernan MA, Brumback B. Marginal structural models and causal inference in epidemiology. Epidemiology. 2000;11(5):550-60.

27. Brookhart MA, Wyss R, Layton JB, Sturmer T. Propensity score methods for confounding control in nonexperimental research. Circ Cardiovasc Qual Outcomes. 2013;6(5):604-11.

28. Rothman KJ. Using regression models in epidemiologic analysis. In: Epidemiology: An Introduction. 2nd ed. New York: Oxford University Press; 2012:211-34.

29. Jones AM. Models for Health Care. Oxford: Oxford University Press; 2011.

30. Li Z, Mahendra G. Using "recycled predictions" for computing marginal effects. Paper presented at: SAS Global Forum 2010; April 11-14, 2010; Seattle, WA. Available at: support.sas.com/resources/papers/proceedings10/272-2010.pdf. Accessed July 26, 2018.

31. Mullahy J. Much ado about two: reconsidering retransformation and the two-part model in health econometrics. J Health Econ. 1998;17(3):247-81.

32. Olender SA, Taylor BS, Wong M, Wilkin TJ. CROI 2015: Advances in Antiretroviral Therapy. Top Antivir Med. 2015;23(1):28-45.

33. Foglia E, Bonfanti P, Rizzardini G, et al. Cost-utility analysis of lopinavir/ritonavir versus atazanavir + ritonavir administered as first-line therapy for the treatment of HIV infection in Italy: from randomised trial to real world. PLoS One. 2013;8(2):e57777.

34. Schackman BR, Scott CA, Walensky RP, Losina E, Freedberg KA, Sax PE. The cost-effectiveness of HLA-B*5701 genetic screening to guide initial antiretroviral therapy for HIV. AIDS. 2008;22(15):2025-33.
35. Jouquet G, Bygrave H, Kranzer K, et al. Cost and cost-effectiveness of switching from d4T or AZT to a TDF-based first-line regimen in a resourcelimited setting in rural Lesotho. J Acquir Immune Defic Syndr. 2011;58(3):e68-74.

36. Juday T, Correll T, Anene A, Broder MS, Ortendahl J, Bentley T. Costeffectiveness of the once-daily efavirenz/emtricitabine/tenofovir tablet compared with the once-daily elvitegravir/cobicistat/emtricitabine/tenofovir tablet as first-line antiretroviral therapy in HIV-infected adults in the U.S Clinicoecon Outcomes Res. 2013;5:437-45.

37. Backus LI, Boothroyd DB, Phillips BR, et al. National quality forum performance measures for HIV/AIDS care: the Department of Veterans Affairs' experience. Arch Intern Med. 2010;170(14):1239-46.

38. Borowsky SJ, Cowper DC. Dual use of VA and non-VA primary care. J Gen Intern Med. 1999;14(5):274-80.

39. Hynes DM, Koelling K, Stroupe K, et al. Veterans' access to and use of Medicare and Veterans Affairs health care. Med Care. 2007;45(3):214-23.

40. Liu CF, Bolkan C, Chan D, Yano EM, Rubenstein LV, Chaney EF. Dual use of VA and non-VA services among primary care patients with depression. J Gen Intern Med. 2009;24(3):305-11.

41. Hynes DM, Koelling K, Stroupe K, et al. Veterans' access to and use of Medicare and Veterans Affairs health care. Med Care. 2007;45(3):214-23.

42. Ross JS, Keyhani S, Keenan PS, et al. Dual use of Veterans Affairs services and use of recommended ambulatory care. Med Care. 2008;46(3):309-16.

43. Lin DY, Feuer EJ, Etzioni R, Wax Y. Estimating medical costs from incomplete follow-up data. Biometrics. 1997;53(2):419-34.

44. Bang H, Tsiatis AS. Estimating medical costs with censored data. Biometrika. 2000;87(2):329-43.

45. Basu A, Manning WG. Estimating lifetime or episode-of-illness costs under censoring. Health Econ. 2010;19(9):1010-28.

46. Schuit SC, van der Klift M, Weel AE, et al. Fracture incidence and association with bone mineral density in elderly men and women: the Rotterdam Study. Bone. 2004;34(1):195-202. 


\section{Health Care Costs in a Cohort of HIV-Infected U.S. Veterans Receiving Regimens Containing Tenofovir Disoproxil Fumarate/Emtricitabine}

\section{APPENDIX A Unadjusted Means and Standard Deviations for Overall, Renal-Specific, and Bone-Specific}

Quarterly Costs and Use

\begin{tabular}{l|c|c|c|c}
\hline & EFV & All Non-EFV & EVG/c & \\
\hline $\mathbf{N}$ & 4,172 & 3,050 & 233 & \\
\hline Quarters & 5.6 & 4.0 & 3.0 & \\
\hline Patient-quarters & 23,189 & 12,073 & 707 & \\
\hline
\end{tabular}

Overall

\begin{tabular}{|c|c|c|c|c|c|c|c|c|c|c|}
\hline \multicolumn{11}{|l|}{ VA cost, $\$$} \\
\hline Outpatient & 2,612 & $(3,619)$ & 2,999 & $(4,737)$ & 3,201 & $(4,258)$ & 3,222 & $(4,558)$ & 2,971 & $(4,778)$ \\
\hline Inpatient & 1,927 & $(11,639)$ & 2,736 & $(17,995)$ & 4,979 & $(45,276)$ & 3,752 & $(20,487)$ & 2,525 & $(14,267)$ \\
\hline Radiology & 215 & $(1,031)$ & 271 & $(2,278)$ & 276 & $(1,218)$ & 298 & $(1,477)$ & 269 & $(2,371)$ \\
\hline Pharmacy & 2,444 & $(6,625)$ & 3,234 & $(5,183)$ & 4,472 & $(4,499)$ & 3,814 & $(4,805)$ & 3,116 & $(5,235)$ \\
\hline Total & 6,983 & $(14,361)$ & 8,969 & $(19,988)$ & 12,652 & $(46,960)$ & 10,788 & $(22,318)$ & 8,612 & $(16,524)$ \\
\hline \multicolumn{11}{|l|}{ VA use } \\
\hline Outpatient encounters & 17.5 & $(26.77)$ & 21.29 & (32.08) & 24.80 & (39.03) & 27.11 & $(43.01)$ & 20.70 & $(30.70)$ \\
\hline Inpatient days & 1.35 & $(7.55)$ & 2.08 & $(9.72)$ & 1.91 & $(7.88)$ & 3.13 & (12.79) & 2.02 & $(9.60)$ \\
\hline \multicolumn{11}{|l|}{ Renal } \\
\hline \multicolumn{11}{|l|}{ VA cost, $\$$} \\
\hline Outpatient & 10 & $(230)$ & 10 & $(208)$ & 11 & (103) & 7 & $(100)$ & 10 & $(218)$ \\
\hline Inpatient & 38 & $(1,451)$ & 40 & $(1,032)$ & 53 & $(1,103)$ & 81 & $(1,278)$ & 36 & $(1,010)$ \\
\hline Radiology & 9 & (9) & 0 & (12) & 0 & $(0)$ & 0 & (0) & 0 & (12) \\
\hline Pharmacy & 2 & (14) & 3 & (166) & 1 & (4) & 2 & (8) & 3 & $(177)$ \\
\hline Total & 48 & $(1,483)$ & 49 & $(1,084)$ & 64 & $(1,179)$ & 88 & $(1,366)$ & 46 & $(1,057)$ \\
\hline \multicolumn{11}{|l|}{ VA use } \\
\hline Outpatient encounters & 0.06 & $(0.60)$ & 0.06 & $(0.78)$ & 0.10 & $(1.61)$ & 0.12 & $(1.25)$ & 0.06 & $(0.65)$ \\
\hline Inpatient days & 0.09 & $(1.34)$ & 0.09 & $(1.45)$ & 0.17 & $(1.26)$ & 0.30 & $(3.64)$ & 0.08 & $(1.20)$ \\
\hline \multicolumn{11}{|l|}{ Bone } \\
\hline \multicolumn{11}{|l|}{ VA cost, $\$$} \\
\hline Outpatient encounters & 11 & $(502)$ & 8 & (311) & 4 & (52) & 51 & $(1,108)$ & 6 & (179) \\
\hline Inpatient & 20 & $(1,255)$ & 15 & $(751)$ & 0 & $(0)$ & 78 & $(1,367)$ & 12 & $(720)$ \\
\hline Radiology & 0.04 & $(5.64)$ & 0.11 & (11) & 0 & (0) & 0 & (0) & 0.13 & (12) \\
\hline Pharmacy & 0.45 & (16) & 0.53 & (16) & 0.04 & $(0.44)$ & 0.02 & $(0.54)$ & 0.60 & (17) \\
\hline Total & 32 & $(1,373)$ & 24 & (828) & 4 & $(52)$ & 129 & $(1,858)$ & 19 & (745) \\
\hline \multicolumn{11}{|l|}{ VA use } \\
\hline Outpatient encounters & 0.01 & $(0.26)$ & 0.02 & $(0.33)$ & 0.03 & $(0.38)$ & 0.07 & $(0.92)$ & 0.02 & $(0.24)$ \\
\hline Inpatient days & 0.01 & $(0.72)$ & 0.03 & $(1.25)$ & 0.11 & $(2.00)$ & 0.01 & $(0.24)$ & 0.03 & (1.22) \\
\hline
\end{tabular}

${ }^{a}$ Costs are in 2015 U.S. dollars.

$E F V=$ efavirenz; $E V G / c=$ elvitegravir/cobicistat; $P I=$ protease inhibitor (atazanavir, darunavir, or lopinavir); $R P V=$ rilpivirine; $R T V=$ ritonavir; $S D=$ standard deviation; $V A=$ Veterans Affairs. 


\section{Health Care Costs in a Cohort of HIV-Infected U.S. Veterans Receiving Regimens Containing Tenofovir Disoproxil Fumarate/Emtricitabine}

APPENDIX B Adjusted Results from IPT-Weighted 2-Part Model for Overall, Renal-Specific, and Bone-Specific Inpatient Quarterly Costs and Use and Radiology Costs

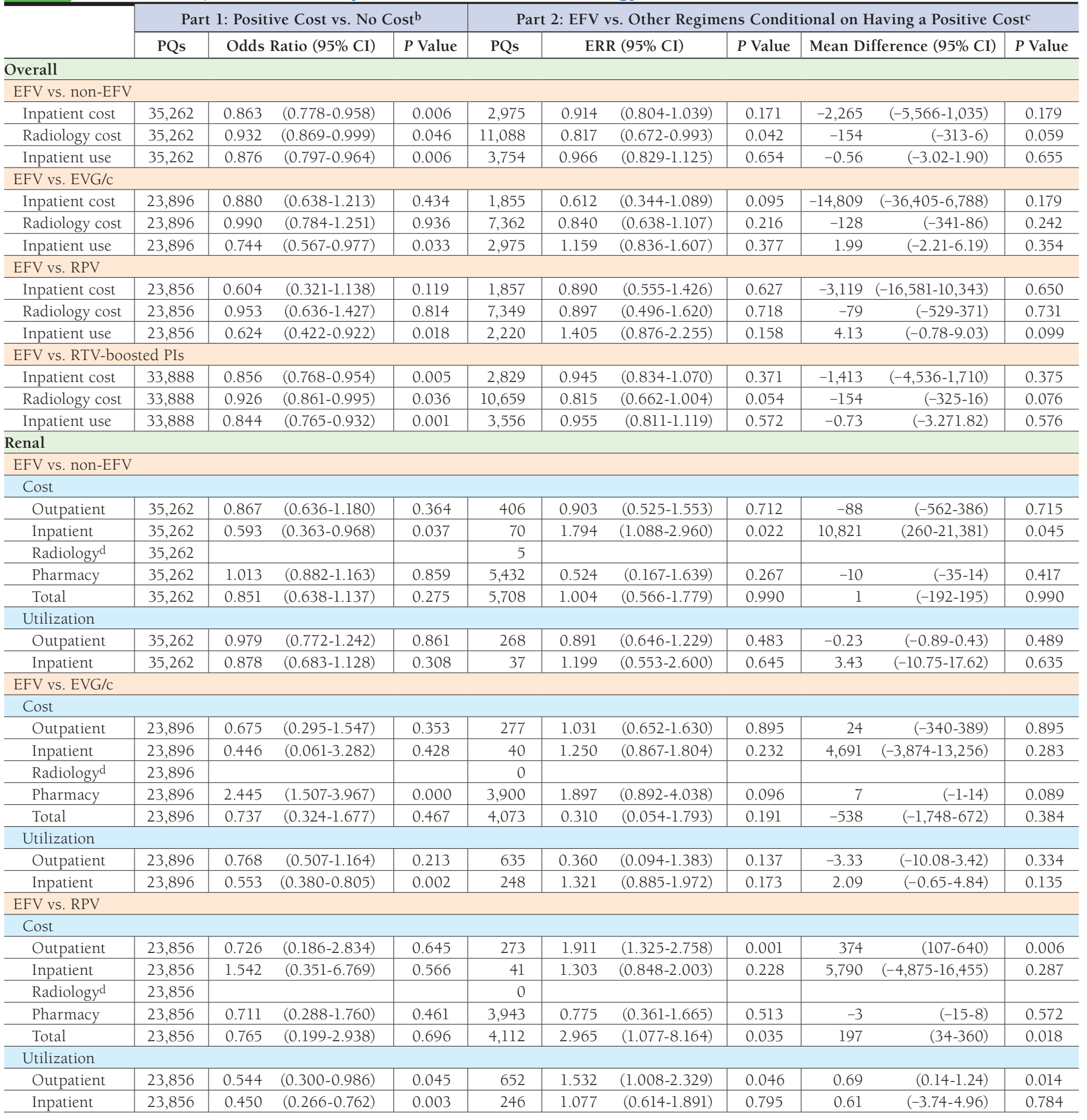




\section{Health Care Costs in a Cohort of HIV-Infected U.S. Veterans Receiving Regimens Containing Tenofovir Disoproxil Fumarate/Emtricitabine}

APPENDIX B Adjusted Results from IPT-Weighted 2-Part Model for Overall, Renal-Specific, and Bone-Specific Inpatient Quarterly Costs and Use and Radiology Costs ${ }^{\mathrm{a}}$ (continued)

\begin{tabular}{|c|c|c|c|c|c|c|c|c|c|}
\hline & \multicolumn{4}{|c|}{ Part 1: Positive Cost vs. No Cost ${ }^{\mathrm{b}}$} & \multicolumn{5}{|c|}{ Part 2: EFV vs. Other Regimens Conditional on Having a Positive Cost ${ }^{\mathrm{c}}$} \\
\hline & PQs & Odds & Ratio (95\% CI) & $P$ Value & PQs & ERR $(95 \% \mathrm{CI})$ & $P$ Value & Mean Difference (95\% CI) & $P$ Value \\
\hline \multicolumn{10}{|c|}{ EFV vs. RTV-boosted PIs } \\
\hline \multicolumn{10}{|c|}{ Cost } \\
\hline Outpatient & 33,888 & 0.856 & $(0.620-1.181)$ & 0.343 & 390 & $(0.521-1.614)$ & 0.764 & $(-573-422)$ & 0.767 \\
\hline Inpatient & 33,888 & 0.599 & $(0.360-0.999)$ & 0.049 & 65 & $(1.080-2.937)$ & 0.024 & $(431-20,078)$ & 0.041 \\
\hline Radiologyd & 33,888 & & & & 5 & & & & \\
\hline Pharmacy & 33,888 & 0.995 & $(0.863-1.147)$ & 0.946 & 5,245 & $0.499 \quad(0.154-1.618)$ & 0.247 & $(-38-15)$ & 0.409 \\
\hline Total & 33,888 & 0.833 & $(0.617-1.125)$ & 0.234 & 5,509 & $(0.565-1.775)$ & 0.996 & $(-188-189)$ & 0.996 \\
\hline \multicolumn{10}{|l|}{ Utilization } \\
\hline Outpatient & 33,888 & 0.935 & $(0.820-1.066)$ & 0.314 & 871 & $(0.694-1.131)$ & 0.331 & $(-0.83-0.28)$ & 0.338 \\
\hline Inpatient & 33,888 & 0.912 & $(0.808-1.031)$ & 0.140 & 246 & $(0.614-1.891)$ & 0.795 & $(-3.74-4.96)$ & 0.784 \\
\hline \multicolumn{10}{|l|}{ Bone } \\
\hline \multicolumn{10}{|c|}{ EFV vs. non-EFV } \\
\hline \multicolumn{10}{|c|}{ Cost } \\
\hline Outpatient & 35,262 & 0.934 & $(0.656-1.328)$ & 0.703 & 235 & $(0.663-3.287)$ & 0.341 & $522 \quad(-610-1,653)$ & 0.366 \\
\hline Inpatient & 35,262 & 0.813 & $(0.333-1.982)$ & 0.648 & 21 & $(0.711-3.915)$ & 0.240 & $15,435(-12,793-43,664)$ & 0.284 \\
\hline Radiologyd & 35,262 & & & & 4 & & & & \\
\hline Pharmacy & 35,262 & 1.231 & $(0.663-2.284)$ & 0.510 & 293 & $(0.478-2.892)$ & 0.725 & $(-48-68)$ & 0.731 \\
\hline Total & 35,262 & 1.055 & $(0.729-1.527)$ & 0.775 & 523 & $(0.603-3.667)$ & 0.389 & $(-1,093-2,691)$ & 0.408 \\
\hline \multicolumn{10}{|l|}{ Utilization } \\
\hline Outpatient & 35,262 & 0.941 & $(0.803-1.103)$ & 0.453 & 268 & $(0.646-1.229)$ & 0.483 & $(-0.89-0.43)$ & 0.489 \\
\hline Inpatient & 35,262 & 0.512 & $(0.240-1.093)$ & 0.084 & 37 & $(0.553-2.600)$ & 0.645 & $(-10.75-17.62)$ & 0.635 \\
\hline \multicolumn{10}{|l|}{ EFV vs. EVG/c } \\
\hline \multicolumn{10}{|l|}{ Cost } \\
\hline Outpatient & 23,896 & 0.663 & $(0.272-1.619)$ & 0.367 & 152 & $(1.480-5.852)$ & 0.002 & $(23-2,532)$ & 0.046 \\
\hline Inpatient & 23,896 & & & & 13 & & & & \\
\hline Radiologyd & 23,896 & & & & 0 & & & & \\
\hline Pharmacy & 23,896 & 0.844 & $(0.200-3.562)$ & 0.818 & 193 & $15.078 \quad(8.496-26.759)$ & 0.000 & (29-185) & 0.007 \\
\hline Total & 23,896 & 0.835 & $(0.306-2.283)$ & 0.726 & 337 & $7.786 \quad(2.992-20.267)$ & 0.000 & $(431-5,928)$ & 0.023 \\
\hline \multicolumn{10}{|l|}{ Utilization } \\
\hline Outpatient & 23,896 & 0.803 & $(0.300-2.152)$ & 0.663 & 176 & $(0.384-2.267)$ & 0.879 & $(-1.97-1.70)$ & 0.882 \\
\hline Inpatient & 23,896 & 0.265 & $(0.058-1.205)$ & 0.086 & 19 & $(0.303-1.912)$ & 0.561 & $(-30.46-17.77)$ & 0.606 \\
\hline \multicolumn{10}{|l|}{ EFV vs. RPV } \\
\hline \multicolumn{10}{|l|}{ Cost } \\
\hline Outpatient & 23,856 & 0.851 & $(0.293-2.473)$ & 0.767 & 155 & $(0.092-1.706)$ & 0.214 & $(-9,013-3,691)$ & 0.412 \\
\hline Inpatient & 23,856 & 0.138 & $(0.030-0.641)$ & 0.011 & 16 & $(0.686-3.610)$ & 0.284 & $(-7,737-29,782)$ & 0.249 \\
\hline Radiologyd & 23,856 & & & & 0 & & & & \\
\hline Pharmacy & 23,856 & 2.377 & $(0.317-17.857)$ & 0.400 & 187 & $(2.587-7.589)$ & 0.000 & $(9-159)$ & 0.028 \\
\hline Total & 23,856 & 1.512 & $(0.543-4.209)$ & 0.428 & 333 & $(0.058-0.341)$ & 0.000 & $-11,975 \quad(-21,971--1,979)$ & 0.019 \\
\hline \multicolumn{10}{|l|}{ Utilization } \\
\hline Outpatient & 23,856 & 0.680 & $(0.218-2.127)$ & 0.508 & 174 & $(0.133-0.849)$ & 0.021 & $(-10.51-2.13)$ & 0.194 \\
\hline Inpatient & 23,856 & 0.197 & $(0.037-1.049)$ & 0.057 & 18 & $(3.054-7.817)$ & 0.000 & $(3.88-19.22)$ & 0.003 \\
\hline \multicolumn{10}{|c|}{ EFV vs. RTV-boosted PIs } \\
\hline \multicolumn{10}{|c|}{ Cost } \\
\hline Outpatient & 33,888 & 1.007 & (0.691-1.468) & 0.971 & 222 & $(0.881-4.157)$ & 0.101 & $806 \quad(-330-1941)$ & 0.164 \\
\hline Inpatient & 33,888 & 1.118 & $(0.398-3.143)$ & 0.832 & 18 & $(0.584-3.966)$ & 0.391 & $13,678 \quad(-19,471-46,827)$ & 0.419 \\
\hline Radiologyd & 33,888 & & & & 4 & & & & \\
\hline Pharmacy & 33,888 & 1.290 & $(0.673-2.473)$ & 0.443 & 285 & $(0.440-2.336)$ & 0.974 & $(-50-51)$ & 0.974 \\
\hline Total & 33,888 & 1.109 & $(0.750-1.638)$ & 0.605 & 503 & $(0.683-5.136)$ & 0.223 & $(-837-3,180)$ & 0.253 \\
\hline Utilization & & & & & & & & & \\
\hline Outpatient & 33,888 & 0.904 & $(0.576-1.419)$ & 0.661 & 252 & $(0.133-0.849)$ & 0.021 & $(-0.62-0.57)$ & 0.930 \\
\hline Inpatient & 33,888 & 0.608 & $(0.266-1.389)$ & 0.238 & 32 & $(0.436-2.464)$ & 0.936 & $(-16.40-17.81)$ & 0.936 \\
\hline $\begin{array}{l}{ }^{a} \text { Costs are in } 201 \\
{ }^{b} \text { Logistic regressi } \\
{ }^{c} \text { IPT-weighted ge } \\
{ }^{d} \text { Not enough pati } \\
\text { CI = confidence in } \\
\text { (atazanavir, daru }\end{array}$ & $\begin{array}{l}\text { Dollars. } \\
\text { alysis. } \\
\text { ized estim }\end{array}$ & ng equ & $\begin{array}{l}\text { ns analysis. } \\
\text { analyze. } \\
\text { ate ration; E }\end{array}$ & virc & $-c_{t}$ & vir/cobicistat; IPT = in & 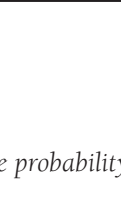 & of treatment; $P I=$ prote & \\
\hline
\end{tabular}

